\title{
EVALUATION OF TWO EGYPTIAN COTTON CULTIVARS IN UPPER EGYPT
}

(Received:26.4.2013)

\author{
By \\ H. A. Idris and S. M. Seyam \\ Cotton Research Institute, Agricultural Research Center, Giza, Egypt.
}

\begin{abstract}
The goal of the present study was suggesting an evaluation of cotton using the least unit of the latin square design ( 2 x2). Two Egyptian cotton (Gossypium barbadense, L.) cultivars, viz., G80 and G90 were used. Cultivars were evaluated at three different locations (Beni Souif, Minia and Assuit) in Upper Egypt during 2010 and 2011 seasons except for Assuit in 2010 season. The studied traits were seed and lint yield, boll components (dry weight, seed cotton, lint cotton, seeds weight and number of seeds), indices (harvest, seed, lint and lint percentage) and fiber properties (fiber length, micronaire reading and Pressely index). Five of $(2 \times 2)$ latin square designs were used in individual locations. The data of five $(2 \times 2)$ latin square design were used together to produce cross over designs. G90 significantly surpassed G80 with respect to seed cotton yield in Beni Souif. In both locations, lint percentage of G80 was greater than the G90. In contrast, harvest index of G90 was greater than G80 since it had the lowest value of dry weight per boll than 80 in both locations. On the other hand, the difference between cultivars with respect to the number of seeds per boll was slight except at Assuit where the differences were significant. The results of fiber properties in both locations revealed that G80 had the longest fiber length compared with G90 due to genetic differences between them and it gave a high micronaire value followed by G90 due to coarseness of fibers. The results of multiple regression revealed that the effects of dry weight per boll, the number of seeds per boll and seed index on seed and lint cotton yield were strong for G80. Results of the present study is important for the regional program to evaluate cotton genotypes.
\end{abstract}

Key words: cotton, genotypes, locations, statistical analysis.

\section{INTRODUCTION}

Experimental units in a latin square design are organized into two groups referred to as rows and columns with regard to the organization of data in a two-way table. Each treatment is assigned the same number of times (usually once) within each group so that differences between groups are not due to treatment effects. At least as many replications are required as there are treatments. Latin squares are usually not practical with more than eight treatments. Only when both rows and columns vary appreciably, will the latin square design improve the detection of treatment differences over the randomized complete block (Little and Hills, 1978).

Abou-Tour et al. (1996) evaluated five Egyptian cotton cultivars, viz., G85, G3, G80, Dendera and G75 at three locations in Upper Egypt ( Fayoum, Assuit and Sohag) using a (5 x 5) latin square design in each location. Results revealed significant differences among cultivars with respect to lint cotton yield, seed index, lint percentage and fiber length in the individual locations. In contrast, non-significant variation due to cultivars was recorded for boll weight.

Awad et al. (2004) evaluated two cultivars G90 and G83 with respect to yield and fiber properties in Upper Egypt (Assuit and Sohag). The results showed that G90 gave 5\% higher yields (seed and lint) than G83. It slightly surpassed G83 for boll weight and gave the same range of lint percentage of G83. Fiber quality for G90 was nearly the same for the long staple cotton group in Upper Egypt.

Idris et al. (2011) evaluated four genotypes using $(4 \mathrm{x} 4)$ latin square design at four locations through two seasons. A compressed latin square design was used to estimate variances of locations and genotypes. The data of each location (two seasons) were considered column and each cell of the design included eight readings. Statistical analysis of compressed was similar to analysis of simple latin square for more than one observation per experimental unit. 
Idris (2012) evaluated two groups of cotton in different zones. The first group was evaluated at two locations in the Delta using a (4 x 4 ) latin square design. The second group was evaluated in the two locations in Upper Egypt using (4 x 4 ) latin square design. Analysis of multiple latin square designs was used to estimate the variance among genotypes in different zones. The data of both the Delta and Upper Egypt locations (4 x 4) were used together to produce a latin square design $(8 \times 8)$. Statistical analysis of the multiple design was similar to the analysis of the simple latin square design.

Researchers need a statistical measure to evaluate genotypes under different locations when the number of treatments is small. Thus, the final goal was to study the possibility of suggesting an evaluation of cotton using the least unit of latin square design $(2 \times 2)$.

\section{MATERIALS AND METHODS}

Five $(2 \times 2)$ latin square designs were carried out at three different locations (Beni Souif, Minia and Assuit) in Upper Egypt during 2010 and 2011 seasons except for Assuit in 2010 season. Latin square design followed was according to Cochran and Cox (1950), Federer (1955), Snedecor and Cochran (1967) and Gomez and Gomez (1984), Table (1).

\subsection{Statistical analysis}

\subsubsection{Analysis of the least unit of latin square design}

For the only $(2 \times 2)$ latin square design, this is zero degrees of freedom associated with the residual sum of squares. Thus, the data of five (2x2) latin square design were used together to produce cross over designs (Table 2). Statistical analysis (Table 3) was straightforward as Bailey (1994), Roger (1994) and Mcpherson (2001). The cultivar means were compared by the least significant difference (L.S.D.) test as given by Steel and Torrie (1980). All comparisons were done at 0.05 level of significance.

\subsubsection{Multiple regression}

The analysis of multiple regression was used to estimate the effect of boll components (x) and indices ( $\mathrm{x}$ ) on both seed and lint cotton yield (y) in the three locations. Statistical analysis was straightforward as Little and Hills (1978), Fowler et al. (1998) and Sing and Narayanan (2000).

\section{RESULTS AND DISCUSSION}

The analysis of variance of the data from individual locations revealed the presence of significant columns, (partitioning columns to squares and columns within squares), rows and genotypes (Table 4).

Table (1) : Layout of five ( $2 \times 2)$ latin square designs in individual locations.

\begin{tabular}{|c|c|c|c|c|c|c|c|c|c|}
\hline \multicolumn{2}{|c|}{ Square (1) } & \multicolumn{2}{|c|}{ Square (2) } & \multicolumn{2}{|c|}{ Square (3) } & \multicolumn{2}{|c|}{ Square (4) } & \multicolumn{2}{|c|}{ Square (5) } \\
\hline A & B & $\mathrm{A}$ & B & B & A & B & A & $\mathrm{A}$ & B \\
\hline B & $\mathrm{A}$ & B & $\mathrm{A}$ & $\mathrm{A}$ & B & $\mathrm{A}$ & B & B & $\mathrm{A}$ \\
\hline
\end{tabular}

Table (2): Layout of cross over designs in individual locations.

\begin{tabular}{|c|c|c|c|c|c|c|c|c|c|}
\hline $\mathrm{A}$ & $\mathrm{B}$ & $\mathrm{A}$ & $\mathrm{B}$ & $\mathrm{B}$ & $\mathrm{A}$ & $\mathrm{B}$ & $\mathrm{A}$ & $\mathrm{A}$ & $\mathrm{B}$ \\
\hline $\mathrm{B}$ & $\mathrm{A}$ & $\mathrm{B}$ & $\mathrm{A}$ & $\mathrm{A}$ & $\mathrm{B}$ & $\mathrm{A}$ & $\mathrm{B}$ & $\mathrm{B}$ & $\mathrm{A}$ \\
\hline
\end{tabular}

The materials used in this study were two Egyptian cotton (Gossypium barbadense, L.) cultivars, viz., G80 and G90. Cultivars were evaluated for seed cotton yield (S.C.Y.) and lint cotton yield (L.C.Y.) in kentar / feddan. One sample of 50 bolls was obtained from each plot to estimate boll components (dry weight $\mathrm{g}$, seed cotton $\mathrm{g}$, lint cotton $\mathrm{g}$, seeds weight $\mathrm{g}$ and number of seeds), indices (harvest, seed, lint and lint percentage) and fiber properties (fiber length $(\mathrm{mm})$, micronaire reading and Pressely index). The lint cotton samples were tested by the Cotton Research Laboratories, Cotton Research Institute.
Table (3): Analysis of variance of cross over designs.

\begin{tabular}{|l|c|}
\hline \multicolumn{1}{|c|}{ Source of variation } & df \\
\hline Columns & $\mathrm{r}-1$ \\
Rows & $\mathrm{t}-1$ \\
Genotypes & $\mathrm{t}-1$ \\
Experimental error & $(\mathrm{t}-1)(\mathrm{r}-2)$ \\
\hline Total & $\mathrm{t} \mathrm{r}-1$ \\
\hline
\end{tabular}

\subsection{Analysis of cross over design}

\subsubsection{Beni Souif location}

In the first season, significant variation due to genotypes was observed for seed cotton yield, dry 
Table (4): Mean square of yield, boll components, indices and fiber properties.

\begin{tabular}{|c|c|c|c|c|c|c|}
\hline \multicolumn{7}{|c|}{ Beni Souif (2010 Season) } \\
\hline & & \multicolumn{2}{|c|}{ Yield } & \multicolumn{3}{|c|}{ Fiber properties } \\
\hline Source of variation & df & Seed & Lint & Length & Micronaire & Pressely \\
\hline Columns & 9 & $2.41 * *$ & $3.71 * *$ & 0.454 & 0.018 & 0.690 \\
\hline Squares (S) & & 1.62 & 2.30 & 0.528 & 0.029 & 0.718 \\
\hline Columns within $(S)$ & 5 & $3.05^{* *}$ & $4.77 * *$ & 0.393 & 0.009 & 0.669 \\
\hline Rows & 1 & 1.76 & 1.93 & $6.85^{* *}$ & 0.200 & 0.012 \\
\hline Genotypes & 1 & $2.87 *$ & 1.10 & 1.11 & $0.512 *$ & 0.180 \\
\hline Experimental error & 8 & 0.446 & 0.629 & 0.249 & 0.092 & 1.02 \\
\hline Total & 19 & \multicolumn{5}{|c|}{ Boll Components } \\
\hline Source of variation & df & Dry weight & $\begin{array}{l}\text { Seed } \\
\text { cotton }\end{array}$ & Lint cotton & $\begin{array}{l}\text { Seeds } \\
\text { weight }\end{array}$ & $\begin{array}{l}\text { No. } \\
\text { seeds }\end{array}$ \\
\hline Columns & 9 & $0.009 * *$ & 0.014 & 0.002 & 0.005 & 0.719 \\
\hline Squares (S) & 4 & $0.013^{* *}$ & 0.028 & 0.004 & 0.011 & 1.11 \\
\hline Columns within $(S)$ & 5 & $0.006^{*}$ & 0.003 & 0.001 & 0.001 & 0.403 \\
\hline Rows & 1 & 0.005 & 0.012 & 0.004 & 0.002 & 1.49 \\
\hline Genotypes & 1 & $0.164 * *$ & 0.029 & 0.001 & 0.035 & 2.97 \\
\hline Experimental error & 8 & 0.001 & 0.036 & 0.007 & 0.012 & 1.69 \\
\hline Total & 19 & \multicolumn{5}{|c|}{ Indices } \\
\hline Source of variation & df & Harvest & Seed & Lint & Lint percent & \\
\hline Columns & 9 & 0.042 & 0.273 & 0.129 & 0.255 & \\
\hline Squares (S) & 4 & 0.054 & 0.347 & 0.206 & \multirow{2}{*}{$\begin{array}{l}0.166 \\
0.324\end{array}$} & \\
\hline Columns within $(S)$ & 5 & 0.032 & 0.214 & 0.067 & & \\
\hline Rows & 1 & 0.011 & 0.014 & 0.001 & 0.716 & \\
\hline Genotypes & 1 & $1.52 * *$ & 0.001 & $0.627 *$ & $13.61 * *$ & \\
\hline Experimental error & 8 & 0.022 & 0.109 & 0.071 & 0.157 & \\
\hline Total & 19 & \multicolumn{5}{|c|}{2011 Season } \\
\hline & & \multicolumn{2}{|c|}{ Yield } & \multicolumn{3}{|c|}{ Fiber properties } \\
\hline Source of variation & df & Seed & Lint & Length & Micronaire & Pressely \\
\hline Columns & 9 & $1.49 *$ & 2.50 & 0.372 & 0.050 & 0.147 \\
\hline Squares (S) & 4 & 0.973 & 1.97 & 0.151 & 0.006 & 0.088 \\
\hline Columns within $(S)$ & 5 & $1.90^{*}$ & 2.92 & 0.549 & 0.085 & 0.193 \\
\hline Rows & 1 & $3.26^{*}$ & $5.13^{*}$ & 0.049 & 0.162 & 0.005 \\
\hline Genotypes & 1 & $4.14^{*}$ & 4.35 & $6.96 * *$ & 0.098 & 0.041 \\
\hline Experimental error & 8 & 0.371 & 0.830 & 0.165 & 0.068 & 0.212 \\
\hline Total & 19 & \multicolumn{5}{|c|}{ Boll Components } \\
\hline Source of variation & df & Dry weight & $\begin{array}{l}\text { Seed } \\
\text { cotton }\end{array}$ & Lint cotton & $\begin{array}{l}\text { Seeds } \\
\text { weight }\end{array}$ & $\begin{array}{l}\text { No. } \\
\text { seeds }\end{array}$ \\
\hline Columns & 9 & 0.007 & 0.051 & 0.011 & 0.016 & 1.91 \\
\hline Squares $(\mathbf{S})$ & 4 & 0.003 & 0.100 & 0.020 & 0.031 & 4.17 \\
\hline Columns within $(S)$ & 5 & 0.010 & 0.012 & 0.003 & 0.004 & 0.108 \\
\hline Rows & 1 & 0.043 & 0.381 & 0.068 & 0.128 & 10.18 \\
\hline Genotypes & 1 & $0.102 *$ & 0.002 & 0.001 & 0.004 & 1.32 \\
\hline Experimental error & 8 & 0.009 & 0.135 & 0.024 & 0.046 & 4.21 \\
\hline Total & 19 & \multicolumn{5}{|c|}{ Indices } \\
\hline Source of variation & df & Harvest & Seed & Lint & Lint percent & \\
\hline Columns & 9 & 0.050 & 0.309 & 0.195 & 0.276 & \\
\hline Squares (S) & 4 & 0.034 & 0.490 & 0.212 & 0.213 & \\
\hline Columns within $(S)$ & 5 & 0.064 & 0.164 & 0.181 & 0.327 & \\
\hline Rows & 1 & 0.001 & 0.025 & 0.025 & 0.013 & \\
\hline Genotypes & 1 & $0.874 * *$ & 0.234 & 0.707 & 2.97 & \\
\hline Experimental error & 8 & 0.049 & 0.254 & 0.293 & 1.04 & \\
\hline Total & 19 & & & & & \\
\hline
\end{tabular}




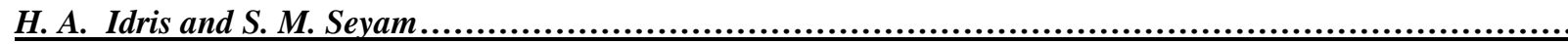

Table (4): Cont. I

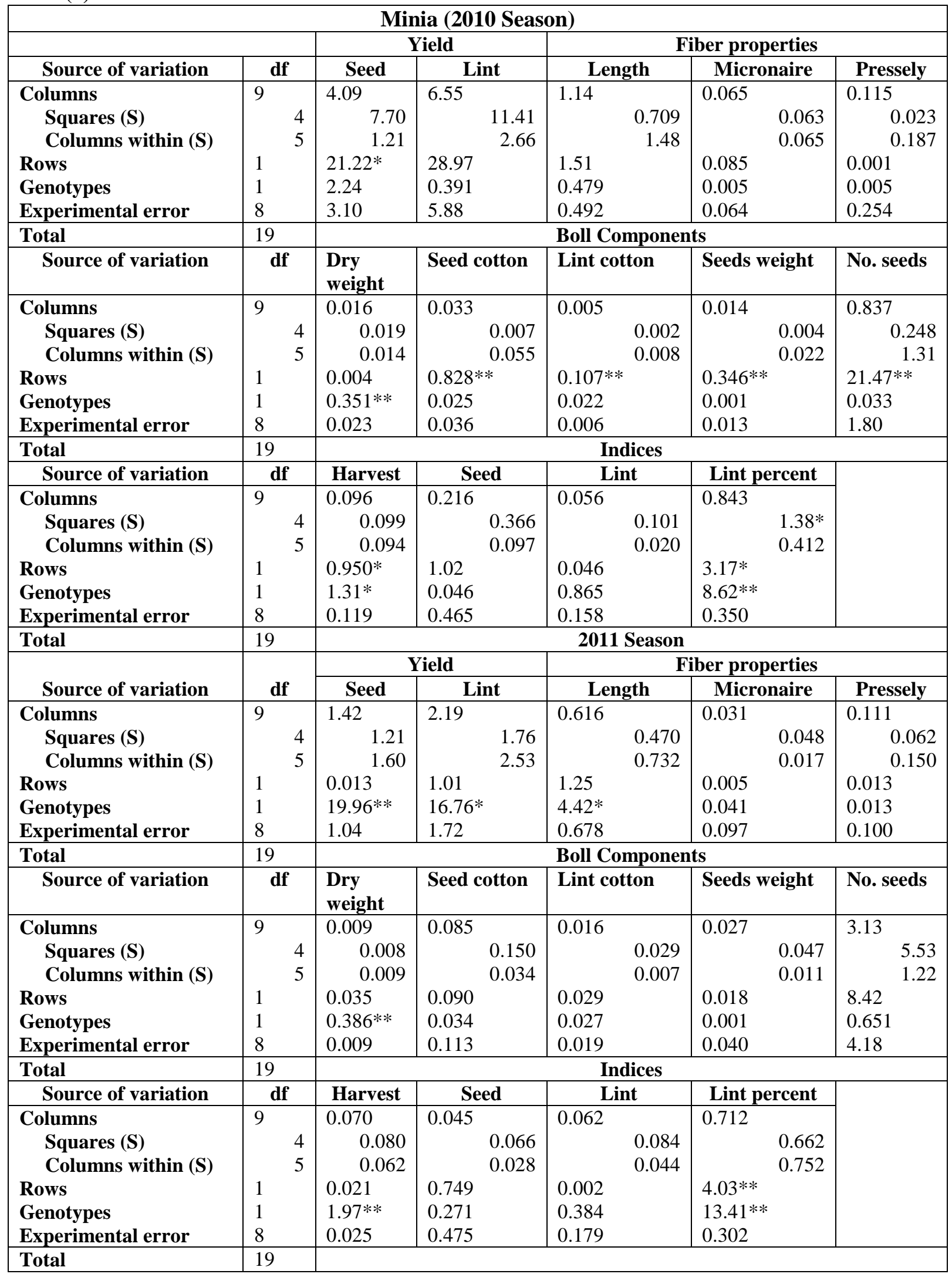


Table (4): Cont. II

\begin{tabular}{|c|c|c|c|c|c|c|}
\hline \multicolumn{7}{|c|}{ Assuit (2011 Season) } \\
\hline & & \multicolumn{2}{|c|}{ Yield } & \multicolumn{3}{|c|}{ Fiber properties } \\
\hline Source of variation & df & Seed & Lint & Length & Micronaire & Pressely \\
\hline Columns & 9 & 3.61 & 6.34 & 1.85 & 0.132 & 0.122 \\
\hline Squares (S) & 4 & 2.11 & 3.99 & 2.72 & 0.167 & 0.165 \\
\hline Columns within $(\mathrm{S})$ & 5 & 4.82 & 8.23 & 1.15 & 0.103 & 0.088 \\
\hline Rows & 1 & 0.152 & 0.253 & 1.74 & 0.001 & 0.018 \\
\hline Genotypes & 1 & 4.53 & 3.86 & 0.002 & 0.112 & 0.512 \\
\hline Experimental error & 8 & 3.36 & 5.44 & 0.929 & 0.114 & 0.482 \\
\hline Total & 19 & \multicolumn{5}{|c|}{ Boll Components } \\
\hline Source of variation & df & Dry weight & Seed cotton & Lint cotton & Seeds weight & No. seeds \\
\hline Columns & 9 & 0.009 & 0.053 & 0.008 & 0.021 & $2.12 *$ \\
\hline Squares $(\mathbf{S})$ & 4 & 0.003 & 0.040 & 0.007 & 0.015 & $2.16^{*}$ \\
\hline Columns within $(\mathrm{S})$ & 5 & 0.013 & 0.063 & 0.009 & $0.025^{*}$ & $2.09 *$ \\
\hline Rows & 1 & 0.003 & $0.265 * *$ & $0.043 * *$ & $0.094 * *$ & 0.990 \\
\hline Genotypes & 1 & 0.013 & $0.269 * *$ & $0.021 *$ & $0.143 * *$ & $5.46 * *$ \\
\hline Experimental error & 8 & 0.023 & 0.020 & 0.004 & 0.007 & 0.450 \\
\hline Total & 19 & \multicolumn{5}{|c|}{ Indices } \\
\hline Source of variation & df & Harvest & Seed & Lint & Lint percent & \\
\hline Columns & 9 & 0.081 & 0.591 & $0.201 *$ & 0.324 & \\
\hline Squares (S) & 4 & 0.067 & 0.182 & 0.158 & 0.293 & \\
\hline Columns within $(\mathrm{S})$ & 5 & 0.093 & 0.918 & $0.237^{*}$ & 0.349 & \\
\hline Rows & 1 & 0.432 & $1.61 *$ & $0.471^{*}$ & 0.062 & \\
\hline Genotypes & 1 & $0.730 *$ & 0.615 & 0.051 & $9.22 * *$ & \\
\hline Experimental error & 8 & 0.123 & 0.269 & 0.052 & 0.215 & \\
\hline Total & 19 & & & & & \\
\hline
\end{tabular}

*, ** Significant at 0.05 and 0.01 levels, respectively.

weight per boll, harvest index, lint index, lint percentage and micronaire reading, (Table 4). G90 significantly surpassed G80 with respect to seed cotton yield and harvest index due to the lowest dry weight per boll. In contrast, G80 significantly exceeded G90 in dry weight per boll, lint index, lint percentage and micronaire reading (Table 5).

In the second season, significant variation due to genotypes was recorded for seed cotton yield, dry weight per boll, harvest index and fiber length (Table 4). G90 significantly exceeded G80 with respect to seed cotton yield and harvest index. G80 significantly surpassed G90 for dry weight per boll and fiber length (Table 5).

Results also showed that G90 was the best cultivar with respect to yield since it gave the highest seed cotton yield and harvest index in both seasons. G80 had the highest value for fiber length compared with G90 in 2011 season.Non-significant differences between the two cultivars were observed for boll components except for dry weight per boll in both seasons, indicating that the boll components were similar in the two cultivars except for dry weight per boll (Table 5).

\subsubsection{Minia location}

In the first season, significant variation due to cultivars was observed for dry weight per boll, harvest index, lint index and lint percentage (Table 4). G80 significantly exceeded G90 with respect to dry weight per boll, lint index and lint percentage. G90 significantly surpassed G80 with respect to harvest index since it had the lowest value of dry weight per boll than G80 (Table 5).

In the second season, significant variation due to genotypes was detected for seed cotton yield, dry weight per boll, harvest index, lint percentage and fiber length (Table 4). G90 significantly surpassed G80 with respect to seed and lint yield and harvest index. In contrast, G80 significantly exceeded G90 in fiber length, dry weight per boll and lint percentage (Table 5).

On the other hand, G80 had the highest values of dry weight per boll and lint percentage, significantly surpassed G90 in the two seasons (Table 5).

\subsubsection{Assuit location}

Non-significant variation due to cultivars was observed for seed and lint yield and fiber properties.In contrast, significant variation between cultivars was recorded for boll components and indices except for dry weight per boll,seed and lint index (Table 4).G90 significantly surpassed G80 with respect to seed cotton per boll, lint cotton per boll, seed weight, number of seeds per boll and harvest index, G80 significantly exceeded G90 for lint percentage,(Table 5).

As an explanation of such results, cultivar 
Table (5): Means of yield, boll components, indices and fiber properties.

\begin{tabular}{|c|c|c|c|c|c|}
\hline \multicolumn{6}{|c|}{ Beni Souif (2010 Season) } \\
\hline \multirow[t]{2}{*}{ Genotypes } & \multicolumn{2}{|c|}{ Yield } & \multicolumn{3}{|c|}{ Fiber properties } \\
\hline & Seed & Lint & Length & Micronaire & Pressely \\
\hline G80 & 8.92 & 11.51 & 32.43 & $4.31 *$ & 9.89 \\
\hline G90 & $9.68 *$ & 11.98 & 32.90 & 3.99 & 9.70 \\
\hline L.S.D. & 0.69 & -- & -- & 0.31 & -- \\
\hline \multirow[t]{2}{*}{ Genotypes } & \multicolumn{5}{|c|}{ Boll Components } \\
\hline & Dry weight & Seed cotton & Lint cotton & Seeds weight & No. seeds \\
\hline G80 & $1.06 *$ & 2.37 & 0.97 & 1.40 & 15.49 \\
\hline G90 & 0.88 & 2.45 & 0.96 & 1.49 & 16.26 \\
\hline L.S.D. & 0.03 & -- & -- & -- & -- \\
\hline \multirow[t]{2}{*}{ Genotypes } & \multicolumn{5}{|c|}{ Indices } \\
\hline & Harvest & Seed & Lint & Lint percent & \\
\hline G80 & 2.24 & 9.16 & $6.29 *$ & $40.97 *$ & \\
\hline G90 & $2.79 *$ & 9.15 & 5.93 & 39.33 & \\
\hline L.S.D. & 0.15 & -- & 0.27 & 0.41 & \\
\hline \multirow{3}{*}{ Genotypes } & \multicolumn{5}{|c|}{2011 Season } \\
\hline & \multicolumn{2}{|c|}{ Yield } & \multicolumn{3}{|c|}{ Fiber properties } \\
\hline & Seed & Lint & Length & Micronaire & Pressely \\
\hline G80 & 10.07 & 13.14 & $31.28 *$ & 4.72 & 10.07 \\
\hline G90 & $10.98 *$ & 14.07 & 30.10 & 4.58 & 10.16 \\
\hline L.S.D. & 0.63 & -- & 0.42 & -- & -- \\
\hline \multirow[t]{2}{*}{ Genotypes } & \multicolumn{5}{|c|}{ Boll Components } \\
\hline & Dry weight & Seed cotton & Lint cotton & Seeds weight & No. seeds \\
\hline G80 & $1.03 *$ & 2.51 & 1.04 & 1.47 & 14.18 \\
\hline G90 & 0.89 & 2.53 & 1.03 & 1.50 & 14.69 \\
\hline L.S.D. & 0.10 & -- & -- & -- & -- \\
\hline \multirow[t]{2}{*}{ Genotypes } & \multicolumn{5}{|c|}{ Indices } \\
\hline & Harvest & Seed & Lint & Lint percent & \\
\hline G80 & 2.44 & 10.40 & 7.37 & 41.46 & \\
\hline G90 & $2.86 *$ & 10.18 & 6.99 & 40.69 & \\
\hline L.S.D. & 0.23 & -- & -- & -- & \\
\hline \multicolumn{6}{|c|}{ Minia (2010 Season) } \\
\hline \multirow[t]{2}{*}{ Genotypes } & \multicolumn{2}{|c|}{ Yield } & \multicolumn{3}{|c|}{ Fiber properties } \\
\hline & Seed & Lint & Length & Micronaire & Pressely \\
\hline G80 & 10.24 & 13.44 & 33.15 & 4.25 & 10.07 \\
\hline G90 & 10.91 & 13.87 & 32.84 & 4.22 & 10.10 \\
\hline L.S.D. & -- & -- & -- & -- & -- \\
\hline \multirow[t]{2}{*}{ Genotypes } & \multicolumn{5}{|c|}{ Boll Components } \\
\hline & Dry weight & Seed cotton & Lint cotton & Seeds weight & No. seeds \\
\hline G80 & $1.26 *$ & 2.66 & 1.11 & 1.55 & 17.07 \\
\hline G90 & 0.99 & 2.59 & 1.04 & 1.55 & 17.15 \\
\hline L.S.D. & 0.16 & -- & -- & -- & -- \\
\hline Genotypes & & & Indices & & \\
\hline & Harvest & Seed & Lint & Lint percent & \\
\hline G80 & 2.13 & 9.09 & $6.51 *$ & $41.73 *$ & \\
\hline G90 & $2.64 *$ & 9.00 & 6.09 & 40.42 & \\
\hline L.S.D. & 0.36 & -- & 0.41 & 0.61 & \\
\hline
\end{tabular}


Table (5): Cont. I

\begin{tabular}{|c|c|c|c|c|c|}
\hline \multirow[t]{3}{*}{ Genotypes } & \multicolumn{5}{|c|}{2011 Season } \\
\hline & \multicolumn{2}{|c|}{ yield } & \multicolumn{3}{|c|}{ Fiber properties } \\
\hline & Seed & Lint & Length & Micronaire & Pressely \\
\hline G80 & 12.07 & 15.41 & $31.22 *$ & 4.39 & 9.99 \\
\hline G90 & $14.07 *$ & $17.24 *$ & 30.28 & 4.48 & 9.94 \\
\hline L.S.D. & 1.05 & 1.35 & 0.85 & -- & -- \\
\hline \multirow[t]{2}{*}{ Genotypes } & \multicolumn{5}{|c|}{$\begin{array}{c}\text { Boll Components } \\
\end{array}$} \\
\hline & Dry weight & Seed cotton & Lint cotton & Seeds weight & No. seeds \\
\hline G80 & $1.12 *$ & 2.51 & 1.02 & 1.49 & 15.87 \\
\hline G90 & 0.85 & 2.42 & 0.94 & 1.48 & 15.43 \\
\hline L.S.D. & 0.10 & -- & -- & -- & -- \\
\hline \multirow{2}{*}{ Genotypes } & \multicolumn{5}{|c|}{ Indices } \\
\hline & Harvest & Seed & Lint & Lint percent & \\
\hline G80 & 2.23 & 9.38 & 6.39 & $40.51 *$ & \\
\hline G90 & $2.86 *$ & 9.62 & 6.11 & 38.88 & \\
\hline L.S.D. & 0.16 & -- & -- & 0.57 & \\
\hline \multicolumn{6}{|c|}{ Assuit (2011 Season) } \\
\hline \multirow[t]{2}{*}{ Genotypes } & \multicolumn{2}{|c|}{ vield } & \multicolumn{3}{|c|}{ Fiber properties } \\
\hline & Seed & Lint & Length & Micronaire & Pressely \\
\hline G80 & 7.56 & 9.85 & 31.12 & 4.22 & 9.51 \\
\hline G90 & 8.51 & 10.73 & 31.11 & 4.07 & 9.19 \\
\hline L.S.D. & -- & -- & -- & -- & -- \\
\hline \multirow[t]{2}{*}{ Genotypes } & \multicolumn{5}{|c|}{ Boll Components } \\
\hline & Dry weight & Seed cotton & Lint cotton & Seeds weight & No. seeds \\
\hline G80 & 0.94 & 2.12 & 0.87 & 1.24 & 15.41 \\
\hline G90 & 0.89 & $2.35 *$ & $0.94 *$ & $1.41 *$ & $16.45 *$ \\
\hline L.S.D. & -- & 0.15 & 0.06 & 0.08 & 0.69 \\
\hline \multirow{2}{*}{ Genotypes } & \multicolumn{5}{|c|}{ Indices } \\
\hline & Harvest & Seed & Lint & Lint percent & \\
\hline G80 & 2.29 & 8.07 & 5.60 & $41.39 *$ & \\
\hline G90 & $2.67 *$ & 8.55 & 5.70 & 40.03 & \\
\hline L.S.D. & 0.36 & -- & -- & 0.48 & \\
\hline
\end{tabular}

--: Not significant at .05 level. $*$ : Cultivar significantly surpassed.

differences in cotton yield are primarily due to differences in reproductive sink. Reproductive sink development depends on the occurrence of the first flower, the time interval between successive flowers and the rate of boll growth (Hearn, 1969). On the other hand, Culp and Harrell (1975) reported that maintaining a high lint percentage was necessary to ensure high lint cotton yield.

G80 had the longest fiber length compared with G90 due to genetic differences between them. G80 gave higher micronaire value followed by G90 due to coarseness of fibers.

The results showed that both genetics and locations affected boll components.

In both locations, the difference between cultivars with respect to the number of seeds per boll was slight but at Assuit these differences were significant.

Harrell and Culp (1976) reported that more numbers of seeds per boll were desirable because of the greater amount of surface area for lint production within the boll. Scholl and Miller (1976) found that selection for both greater seed per boll and larger seed would produce a reduction in lint yield.

Harvest index is a compound character since it depends on two primary factors, weight of seed cotton per boll and weight of dry weight per boll. It is expected to vary considerably according to fluctuations of the two factors.

In both locations, harvest index of G90 was greater than G80 due to second factor (dry weight per boll) since it had the lowest value. This may explain the transcend of G80 over G90 and significant differences for dry weight per boll 
Table (6): Mean square of multiple regression of yield (y), boll components $(x)$ and indices $(x)$.

\begin{tabular}{|c|c|c|c|c|c|c|c|c|}
\hline & \multicolumn{8}{|c|}{ Seed cotton yield $(y)$} \\
\hline Boll components $(\mathbf{x})$ & \multicolumn{3}{|c|}{ Beni Souif } & \multicolumn{2}{|c|}{ Minia } & \multicolumn{3}{|c|}{ Assuit } \\
\hline Source of variation & df & G80 & G90 & G80 & G90 & df & G80 & G90 \\
\hline Regression & 5 & 2.17 & 2.09 & 5.12 & 7.38 & 5 & 7.36 & 0.741 \\
\hline Dry weight $\left(\mathbf{x}_{1}\right)$ & 1 & 1.08 & 1.93 & $22.38 * *$ & 9.83 & & 0.090 & 0.998 \\
\hline Seed cotton $\left(x_{2}\right)$ & 1 & 2.94 & 2.01 & 1.35 & 4.85 & & 10.84 & 2.04 \\
\hline Lint cotton $\left(\mathbf{x}_{3}\right)$ & 1 & 0.186 & 2.03 & 1.44 & 13.91 & & 0.644 & 0.009 \\
\hline Seeds weight $\left(\mathrm{x}_{4}\right)$ & 1 & 0.125 & 0.502 & 0.412 & 3.26 & & 25.00 & 0.081 \\
\hline No. Seeds $\left(x_{5}\right)$ & 1 & $6.51 *$ & 3.97 & 0.010 & 5.05 & & 0.218 & 0.578 \\
\hline Residual & 14 & 0.859 & 2.03 & 2.11 & 5.63 & 4 & 4.17 & 0.593 \\
\hline Total & 19 & & & & & 9 & & \\
\hline Indices (x) & \multicolumn{3}{|c|}{ Beni Souif } & \multicolumn{2}{|c|}{ Minia } & \multicolumn{3}{|c|}{ Assuit } \\
\hline Source of variation & df & G80 & G90 & G80 & G90 & df & G80 & G90 \\
\hline Regression & 4 & 2.16 & 1.90 & 5.40 & 9.66 & 4 & 1.69 & 0.626 \\
\hline Harvest $\left(\mathbf{x}_{6}\right)$ & 1 & 2.14 & 3.27 & $12.71 *$ & 13.91 & & 3.52 & 0.433 \\
\hline Seed $\left(\mathbf{x}_{7}\right)$ & 1 & $5.33 *$ & 4.05 & 0.131 & 19.38 & & 0.009 & 0.004 \\
\hline Lint $\left(\mathbf{x}_{8}\right)$ & 1 & 0.494 & 0.103 & 8.18 & 0.602 & & 3.07 & 2.05 \\
\hline Lint percent. $\left(\mathbf{x}_{9}\right)$ & 1 & 0.691 & 0.170 & 0.575 & 4.76 & & 0.167 & 0.017 \\
\hline Residual & 15 & 0.947 & 2.08 & 2.23 & 5.14 & 5 & 9.34 & 0.714 \\
\hline Total & 19 & & & & & 9 & & \\
\hline & \multicolumn{8}{|c|}{ Lint cotton yield $(\mathbf{y})$} \\
\hline Boll components $(\mathbf{x})$ & \multicolumn{3}{|c|}{ Beni Souif } & \multicolumn{2}{|c|}{ Minia } & \multicolumn{3}{|c|}{ Assuit } \\
\hline Source of variation & df & G80 & G90 & G80 & G90 & df & G80 & G90 \\
\hline Regression & 5 & 4.09 & 4.60 & 6.01 & 7.59 & 5 & 12.42 & 1.24 \\
\hline Dry weight $\left(\mathbf{x}_{1}\right)$ & 1 & 2.14 & 1.91 & $27.38 *$ & 6.69 & & 0.180 & 2.47 \\
\hline Seed cotton $\left(x_{2}\right)$ & 1 & 5.44 & 4.83 & 2.20 & 7.11 & & 18.32 & 2.60 \\
\hline Lint cotton $\left(\mathbf{x}_{3}\right)$ & 1 & 1.67 & 9.34 & 0.146 & 10.06 & & 0.623 & 0.125 \\
\hline Seeds weight $\left(\mathrm{x}_{4}\right)$ & 1 & 0.322 & 0.819 & 0.415 & 5.80 & & 42.56 & 0.090 \\
\hline No. Seeds $\left(x_{5}\right)$ & 1 & $10.85^{*}$ & 6.12 & 0.001 & 8.30 & & 0.397 & 0.935 \\
\hline Residual & 14 & 1.43 & 3.31 & 3.63 & 8.93 & 4 & 7.10 & 1.04 \\
\hline Total & 19 & & & & & 9 & & \\
\hline Indices (x) & \multicolumn{3}{|c|}{ Beni Souif } & \multicolumn{2}{|c|}{ Minia } & \multicolumn{3}{|c|}{ Assuit } \\
\hline Source of variation & df & G80 & G90 & G80 & G90 & dff & G80 & G90 \\
\hline Regression & 4 & 4.23 & 4.76 & 5.87 & 10.14 & 4 & 3.00 & 1.18 \\
\hline Harvest $\left(\mathrm{x}_{6}\right)$ & 1 & 3.85 & 6.34 & 16.78 & 13.90 & & 6.14 & 0.244 \\
\hline Seed $\left(\mathbf{x}_{7}\right)$ & 1 & $10.84 *$ & 11.93 & 0.003 & 18.26 & & 0.03 & 0.024 \\
\hline Lint $\left(\mathbf{x}_{8}\right)$ & 1 & 1.92 & 0.371 & 5.57 & 0.101 & & 5.22 & 4.41 \\
\hline Lint percent. $\left(\mathbf{x}_{9}\right)$ & 1 & 0.303 & 0.391 & 1.11 & 8.28 & & 0.62 & 0.020 \\
\hline Residual & 15 & 1.57 & 3.35 & 3.83 & 8.16 & 5 & 15.70 & 1.14 \\
\hline Total & 19 & & & & & 9 & & \\
\hline
\end{tabular}

*, ** Significant at 0.05 and 0.01 levels, respectively.

compared with the first factor (seed cotton per boll).

Lint percentage is a compound character since it depends on two primary factors, weight of lint and weight of seed. It is expected to vary considerably according to fluctuations of the two factors. In both locations lint percentage of G80 was greater than G90. This may explain the transcend of G80 over G90 with lint index.

\subsection{Multiple regression}

The analysis of multiple regression revealed the effect of boll components (x) and indices (x) on both seed and lint cotton yield (y) in the three locations (Table 6).
In the first analysis, when considered the effect of boll components on seed cotton yield exhibited effects of both number of seeds per boll and dry weight per boll were significant with respect to G80 in Beni Souif and Minia, respectively.

In the second analysis, when considered the effect of indices on seed cotton yield revealed that the effects of seed index and harvest index were significant with respect to G80 in Beni Souif and Minia, respectively.

On the other hand, when considered the effect of boll components on lint cotton yield, exhibited effects of both number of seeds per boll and dry 
weight per boll were significant with respect to G80 in Beni Souif and Minia, respectively. Also, the effect of indices on lint cotton yield revealed that the effect of seed index was significant with respect to G80 in Beni Souif.

As an explanation of such results, the effects of dry weight per boll, number of seeds per boll and seed index were strong on seed and lint cotton yield for G80.

In this respect, Idris (2008) in his evaluation of some Egyptian cotton genotypes in the Delta, found that the effect of boll weight and then the additional effect of seed index on seed cotton yield exhibited that the effect of boll weight was significant with respect to G86. On the other hand, when considered the effect of boll weight and then the additional effect of lint percentage on lint cotton yield exhibited that effect of lint percentage was significant with respect to G89 x G86 in the Delta.

\section{REFERENCES}

Abou-Tour H. B., Seyam S. M. and Abd El Rahman L. M. (1996). Analytical study on the economic characters of new and commercial Egyptian cotton. Egypt. J. Agric. Res., 74 (3) : 781-791.

Awad H.Y., Awaad M.M., Mohamed S.A., El Adly H.H., El Ameen T.M., Mohamed A.A. and Eissa A.E. (2004). Giza 90, a new , long staple Egyptian cotton variety for Middle and Upper Egypt. Egyptain Journal of Agricultural Research Center, 82 (3) 1349-1361.

Bailey N. T. (1994). Statistical Methods in Biology. Third Edit. Cambridge University Press, Cambridge, England.

Cochran W.G and Cox G. M. (1950). Experimental Designs. John Wiley and Sons, New York., U.S.A.

Culp T. W. and Harrell D. C. (1975). Influence of lint percentage, boll size and seed size on lint yield of Upland cotton with high fiber strength. Crop Sci. 15 ; 741 - 746.

Federer W. T. (1955). Experimental Design. Theory and Application. The MacMilan Company New York, U.S.A.

Fowler J., Cohen L. and Jarvis P. (1998). Practical
Statistics for Field Biology. Second Edit., John Wiley and Sons, New York, U.S.A.

Gomez K. A. and Gomez A. A. (1984). Statistical Procedures for Agricultural Research. John Wiley and Sons, New York, U.S.A.

Harrell D. C. and Culp T. W. (1976). Effect of yield components on lint yield of Upland cotton with high fiber strength. Crop Sci. 16 : $205-208$.

Hearn A. B. (1969). Growth and performance of cotton in a desert environment. Dry matter production. J. Agric. Sci. 73 : 75 - 86.

Idris H.A. (2008). Evaluation of some Egyptian cotton genotypes in the first and the second picks by Latian square design with a combined analysis. Bull. Fac. Agric., Cairo Univ., 59 (1): $13-23$.

Idris H.A. (2012). Comparison between some cultivars and varieties of Egyptian cotton using different analysis of latin square design. Bull. Fac. Agric., Cairo Univ., 63 (1): 19 - 28.

Idris H.A., El Tahan S.M. and Badr S.S. (2011). Evaluation of some Egyptian cotton genotypes in the Delta using both compressed and collected latin square design. Bull. Fac. Agric., Cairo Univ., 62 (3): 303 - 315.

Little T.M. and Hills F. J. (1978). Agricultural Experimentation Design and Analysis. John Wiley and Sons, New York., U.S.A.

Mcpherson G. (2001). Applying and Interpreting Statistics A Comprehensive Guide. Second Edit., Springer Verlag, New York, Inc. U.S.A.

Roger G. P. (1994). Agricultural Field Experiments Design and Analysis. Marcel Dekker, Inc. New York, U.S.A.

Scholl R. L. and Miller P. A. (1976). Genetic association between yield and fiber strength in Upland cotton. Crop Sci., 16 : 780 -783.

Sing P. and Narayanan S. S. (2000). Biometrical Techniques in Plant Breeding. Second Edit., Ludhiana, New Delhi, India.

Snedecor G W. and Cochran W. G. (1967). Statistical Methods. Iowa State Univ. Press, Ames, Iowa, U. S. A.

Steel R. G. and Torrie J. H. (1980). Principles and Procedures of Statistics. Second Edit., McGraw. Hill. Book Co. New York, U.S.A. 


\section{تقييم صنفين من القطن المصري في الوجه القبلي \\ حاتم أحمد إدريس - سعيد مصطفى صيام \\ معهد بحوث القطن - مركز البحوث الزر اعية ـ الجيزة - مصر}

\section{ملخص}

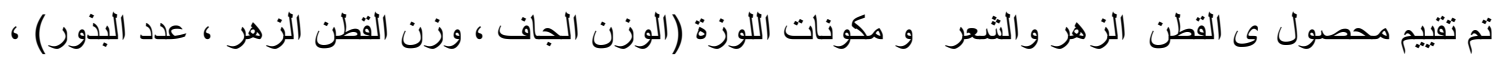

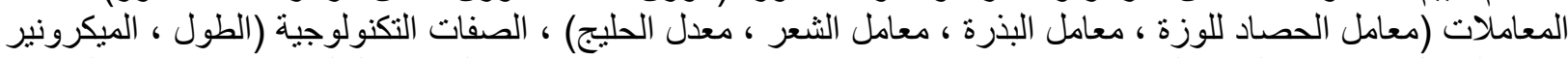

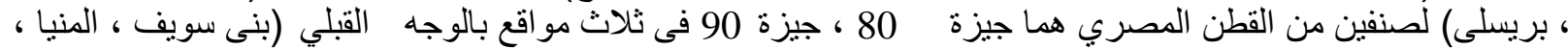

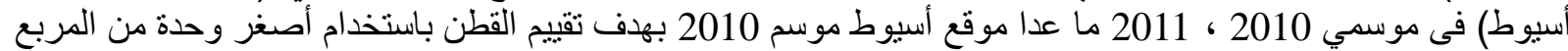
اللاتيني. تم تقييم الصنفين فى خمس مربعات لاتيني (2 x 2) في كل موقع ونم تجميع الخمس مربعات معا لتكوين تصميم .cross over designs

أظهرت النتائج تفوق جيزة 90 معنويا بالنسبة لمحصول القطن الزهر هر في بنى سويف كما تفوق جيزة 80 في معدل

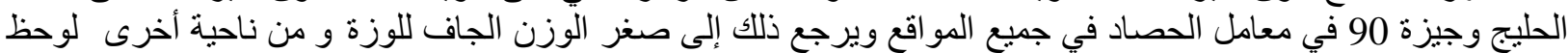

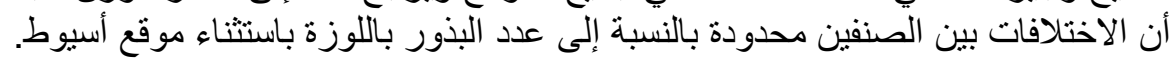

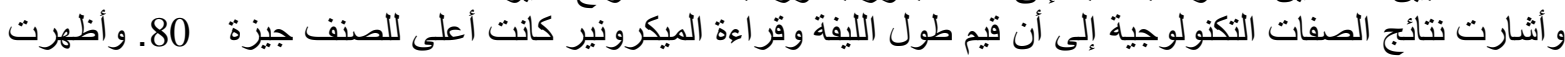

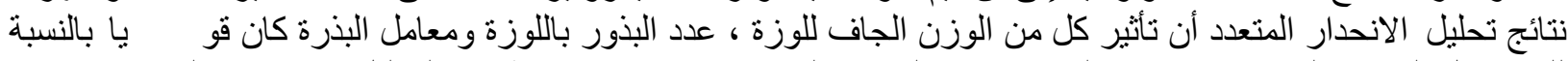
للمحصول الز هر و الثعر. تعتبر هذه الدر اسة مهمة لبر امج التقييم من حيث هدفها وطريقة التحليل الإحصائي المستخدمة. التهان.

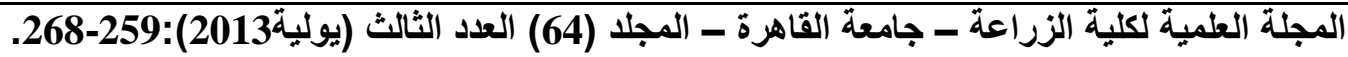

